\title{
Prevalence and trend of emerging and re-emerging arboviral infections in the state of Odisha
}

\author{
Subhra Subhadra ${ }^{1} \cdot$ Jyotsnamayee Sabat $^{1} \cdot$ Bhagirathi Dwibedi $^{2} \cdot$ Sailendra Panda $^{1}$. \\ Madhab Charan Mandal ${ }^{1}$ - Sonalika Rath ${ }^{1} \cdot$ Lal Mohan Ho $^{1} \cdot$ Subrata Kumar Palo $^{1}$. \\ Sanghamitra Pati ${ }^{1} \cdot$ Jyotirmayee Turuk $^{1}$ (i)
}

Received: 23 September 2020/Accepted: 14 July 2021 / Published online: 26 July 2021

(C) Indian Virological Society 2021

\begin{abstract}
Newly emerging or re-emerging infections are posing continuous threat to both public health system and clinical care globally. The emergence of infections especially caused by arboviruses can be linked to several mechanisms which include geographical expansion linked to human development and transportation, global warming, enhanced transmission in peridomestic area and close proximity of human habitations to domestic as well as wild animals. The co-circulation of Dengue, Chikungunya and Zika is a matter of public health priority due to the fact that they are transmitted by the same vector as well as increase in the number of reported cases of severe dengue, postchikungunya chronic joint disease and microcephaly related to Zika virus disease. The study was designed to estimate the prevalence of these arboviral infections in Odisha. About 5198 cases presenting with common clinical symptoms of fever, arthralgia, headache, myalgia and malaise were screened during 2016-2019. A total of $42.2 \%$ patients tested positive for dengue NS1 antigen $(n=4154)$, $30.2 \%$ for dengue $\operatorname{IgM}(\mathrm{n}=2161)$ and $14.3 \%$ for chikungunya $\operatorname{IgM}(\mathrm{n}=1816)$. A total of 1684 samples were subjected to Zika RT-PCR and none was tested positive. Peak in the numbers of dengue/ chikungunya cases was evident in the post-monsoon months of July - October. Circulation of all four serotypes of dengue i.e. DEN 1, 2, 3,
\end{abstract}

Bhagirathi Dwibedi, Jyotirmayee Turuk the authors have contributed equally to this work.

Jyotirmayee Turuk

drjyotirmayeeturuk@gmail.com

1 Virus Research and Diagnostic Laboratory, ICMR - Regional Medical Research Centre, Bhubaneswar, Odisha, India

2 Department of Pediatrics, AIIMS, Bhubaneswar, Odisha, India and 4 was noticed in the state. Molecular investigation of suspected Chik cases in early phases showed circulation of Eastern Central Southern African genotype (E1:226A). There is dearth of knowledge about disease severity during arbovirus co-infections and importance of adequate management of patients at an early stage residing in risk areas. It is the first study in Odisha to study the pattern and status of these three arboviral diseases Dengue, Chikungunya and Zika. The outcome of this study will help in focusing and improvement of existing surveillance systems and vector control tools, as well as on the development of suitable antiviral agents and formulating candidate vaccine.

Keywords Dengue $\cdot$ Chikungunya $\cdot$ Zika $\cdot$ Odisha

\section{Introduction}

An upsurge in emerging and re-emerging arboviral diseases has led to a global concern of unpredictable public health threats. This increased incidence has been attributed largely to geographical expansion in the recent years. Dengue, Chikungunya, Rift Valley fever, Zika, West Nile fever, Japanese encephalitis and Yellow fever among others are all vector-borne viral diseases resulting in morbidity and mortality. With increase in globalization the arboviruses causing dengue, chikungunya, and Zika have spread rapidly throughout the globe, overcoming inter-continental barriers, affecting naïve populations that lack native immunity. Among all emerging arboviral infections, Dengue, caused by Flavivirus belonging to the family Flaviviridae, is the most prevalent. According to the World Health Organization (WHO), dengue is spreading worldwide rapidly, becoming endemic in various inhabited parts of world excluding continental Europe [1]. Chikungunya, 
an alphavirus belonging to the family Togaviridae, is a mosquito-borne re-emerging arboviral disease characterized by febrile illness and severe arthralgia [2, 3]. Zika virus, related to dengue virus also belongs to the genus Flavivirus of family Flaviviridae and is responsible for causing Zika virus disease outbreak which was officially declared by WHO in February 2016 as a "Public Health Emergency of International Concern" (PHEIC) [4]. Dengue virus (DENV), chikungunya virus (CHIKV) and Zika virus (ZIKV) comprise of similar epidemiology, modes of transmission and clinical manifestations especially during the early phases of infection, although complications vary markedly. The transmission cycle of these three arboviruses involves Aedes aegypti, Aedes albopictus species of mosquito [5]. Majority of all infections of dengue, chikungunya and Zika include asymptomatic and mild clinical forms, mostly presenting with similar clinical profile including fever, myalgia, headache, arthralgia, maculopapular rash, lymphadenopathies and retro-orbital pain [6]. Regular occurrence of dengue, re-emergence of chikungunya and emergence of Zika involving a common transmitting vector and similar clinical presentation urges for the need of active surveillance and implementation of control as well as prevention measures. Keeping this in view, the present study was designed to investigate the prevalence and trend of these three vector-borne viral diseases i.e. dengue, chikungunya and Zika in the state of Odisha.

\section{Materials and methods}

\section{Study area and it's geo-climatic environment}

The state of Odisha has a total of thirty districts and lies between the latitudes $17.78^{\circ} \mathrm{N}$ and $22.73^{\circ} \mathrm{N}$, and between longitudes $81.37^{\circ} \mathrm{E}$ and $87.53^{\circ} \mathrm{E}$ on the eastern coast of India. Being a coastal state, the weather is highly influenced by Bay Of Bengal Sea. Climate is tropical in nature resulting in very high environmental temperature ranging between 35 to $40{ }^{\circ} \mathrm{C}$ during summer months of April and May and temperature ranging between 12 to $14{ }^{\circ} \mathrm{C}$ during the winter season. The state experiences south west monsoon during July-September with an average rainfall of $150 \mathrm{~cm}$.

\section{Study design and duration}

This observational study was conducted in regional level Viral Diagnostic and Research Laboratory, RMRC, Bhubaneswar from January, 2016 to December, 2019.

\section{Study participants}

We included patients from all districts of Odisha, as well as from outpatient departments and wards of Govt. Medical Colleges and private hospitals, and outpatient department of RMRC, Bhubaneswar. A total of 5198 samples satisfying the inclusion criteria were included in this study and tested for dengue, chikungunya or Zika. Detailed clinical history of the patients with travel history, if any was collected in a predesigned questionnaire form.

\section{Ethical considerations}

The study was approved by Human Ethical Committee of the Institution following guidelines of Indian Council of Medical Research (ICMR), New Delhi.

\section{Laboratory investigation}

Approximately 2 to $3 \mathrm{ml}$ of blood was collected from each individual patient following strict aseptic precaution. Samples were centrifuged at $3000 \mathrm{rpm}$ for five minutes for separation of serum and tested immediately. In case of delay in processing, sera were stored at a temperature of 2 to $8{ }^{\circ} \mathrm{C}$. Informed consent was obtained from the patients.

\section{(i) Serological}

Depending upon the duration of illness, the sera samples were tested serologically, for detection of NS1 antigen (history of fever $=/<5$ days) or anti DENV IgM antibodies (history of fever $>5$ days). Detection of NS1 antigen was done using DENV Detect NS1 ELISA kit, by In Bios International Inc. USA and IgM anti dengue antibodies were detected by NIV dengue IgM capture kits by NIV, Pune, India. The laboratory tests were performed following the instruction manual provided in the kits. The samples were also subjected to serological diagnosis of chikungunya using NIV CHIK IgM Capture ELISA kits provided by NIV, Pune, India. Samples of patients that tested positive for presence of NS1 antigen and anti dengue IgM antibodies were considered as dengue positive and similarly chik IgM positive samples were considered as confirmed chikungunya cases.

(ii) RT-PCR

The samples which were DENV and CHIKV negative were subjected to molecular detection of ZIKV by RT-PCR followed by viral RNA extraction from samples, using QIAamp Viral RNA mini kit (QIAGEN, Gmbh Hildem,Germany) following manufacturer's instruction. For dengue serotyping RT-PCR assay was performed targeting core 
premembrane (CprM) gene of NS1 positive samples. Based on the size of the PCR products, four dengue serotypes were distinguished as described by Lanciotti et al. [7]. Conventional PCR targeting a region of the gene that encodes for the $\mathrm{E}$ protein was also performed for the detection of the CHIKV [8]. To identify the circulating serotypes in Odisha, genetic characterization of CHIK V isolates was also done [9]. To avoid PCR contamination, prePCR and post-PCR steps were spatially separated. For detection of possible contamination, a no template negative control was included in all the PCR assays. The PCR products were analyzed using $2 \%$ agarose gel, stained with ethidium bromide which was examined under ultraviolet light using gel documentation system (Alpha Imager, USA).

\section{Statistical analysis}

The epidemiological parameters were analysed which included demography, clinical profile of the arboviral diseases and possible outcome. Data generated was analyzed using Microsoft Excel.

\section{Result}

During January, 2016 to December, 2019; a total of 5198 samples suspected for dengue, chikungunya or Zika were received and investigated at regional VRDL, ICMRRMRC, Bhubaneswar. The cases were in all age groups ranging from 3 days to 100 years. Out of total investigated cases, 3132 were male and 2066 were female. Among the 5198 samples investigated, 5100 were tested for dengue, 1816 for chikungunya and 1684 for Zika. The year wise distribution as well as detailed number of DENV, CHIKV and ZIKV is presented in Table 1.

\section{CHIKV and ZIKV}

CHIKV and DENV co-infection was detected $6.36 \%$ cases $(\mathrm{n}=1729)$. Prevalence of DENV, CHIKV and ZIKV during the study period i.e. 2016-2019 has been depicted in Fig. 1.

Comparison of suspected and confirmed cases of dengue, chikungunya and Zika with determinants like gender and age group is shown below in Table 2 .

Highest incidence of dengue was noted in the age group of adults i.e. $19-59$ years i.e. $48.8 \%$ and for chikungunya 60 and above age group i.e. senior adults were most affected (23.1\%).

Geo-climatic distribution of cases under study showed high prevalence in coastal districts $(\mathrm{n}=3682)$ of Odisha as compared to the non-coastal districts $(n=1516)$.

Common clinical presentations noted were fever, arthralgia, headache, myalgia and malaise. Table 3 includes the clinical manifestations associated with laboratory confirmed positive cases of dengue and chikungunya.

Significant seasonal pattern was observed. There appeared to be a peak of dengue/ chikungunya cases in the months of July-October following monsoon. Figure 2 depicts month wise prevalence of dengue/ chikungunya cases during the study period.

Serotyping was done using RTPCR by amplifying a part of core-pre-membrane gene (CprM) which detected presence of all four serotypes 1, 2, 3, 4 of DENV in the state with DEN2 being the major circulating serotype (69\%). Genetic characterization of CHIKV isolates revealed presence of Eastern Central Southern African genotype (E1:226A) in Odisha.

\section{Discussion}

Recent rise in the incidence of arboviral infections, associated clinical complications and severity of cases have made arboviral diseases like dengue, chikungunya and Zika

Table 1 Year wise distribution of suspected /confirmed dengue, chikungunya and Zika cases

\begin{tabular}{|c|c|c|c|c|c|c|}
\hline \multirow[t]{2}{*}{ Year } & \multicolumn{2}{|l|}{ Dengue } & \multicolumn{2}{|l|}{ Chikungunya } & \multicolumn{2}{|l|}{ Zika } \\
\hline & $\begin{array}{l}\text { No. of suspected } \\
\text { cases }\end{array}$ & $\begin{array}{l}\text { No. of confirmed cases } \\
(\%)\end{array}$ & $\begin{array}{l}\text { No. of suspected } \\
\text { cases }\end{array}$ & $\begin{array}{l}\text { No. of confirmed cases } \\
(\%)\end{array}$ & $\begin{array}{l}\text { No. of suspected } \\
\text { cases }\end{array}$ & $\begin{array}{l}\text { No. of confirmed cases } \\
(\%)\end{array}$ \\
\hline 2016 & 742 & $373(50.3)$ & 512 & $48(9.3)$ & 399 & $0(0)$ \\
\hline 2017 & 689 & $371(53.8)$ & 265 & $33(12.4)$ & 178 & $0(0)$ \\
\hline 2018 & 2910 & 1205 (41.4) & 744 & $81(10.9)$ & 931 & $0(0)$ \\
\hline 2019 & 759 & 318 (41.9) & 295 & $97(32.8)$ & 176 & $0(0)$ \\
\hline
\end{tabular}

Assay/s (serological/RT-PCR) employed: Dengue NS1/IgM ELISA, Chik IgM ELISA, RT-PCR for DENV, CHIKV and ZIKV 

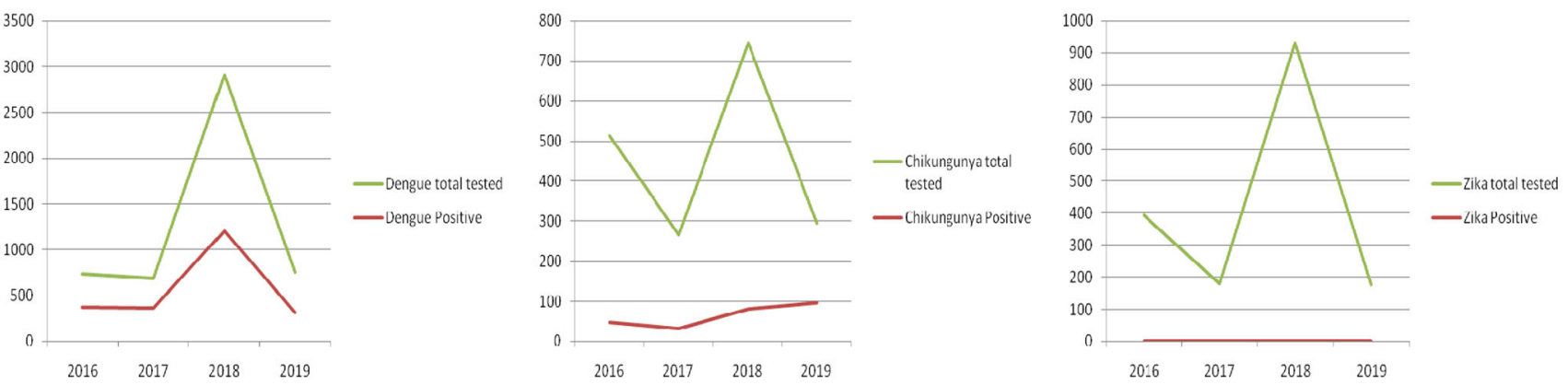

Fig. 1 Year wise prevalence of suspected /confirmed dengue, chikungunya and Zika cases

Table 2 Comparison of suspected and confirmed cases of dengue, chikungunya and Zika

Table 3 Clinical manifestations associated with confirmed cases of dengue and chikungunya

\begin{tabular}{|c|c|c|c|c|c|c|}
\hline \multirow[t]{2}{*}{ Determinants } & \multicolumn{2}{|c|}{ Dengue $(\mathrm{n}=5100)$} & \multicolumn{2}{|c|}{ Chikungunya $(\mathrm{n}=1816)$} & \multicolumn{2}{|c|}{ Zika $(\mathrm{n}=1684)$} \\
\hline & Suspected & Positive $(\%)$ & Suspected & Positive $(\%)$ & Suspected & Positive (\%) \\
\hline \multicolumn{7}{|l|}{ Gender } \\
\hline Male $(n=3132)$ & 3086 & $1367(44.3)$ & 1084 & $134(12.4)$ & 1034 & 0 \\
\hline Female $(n=2066)$ & 2014 & $900(44.7)$ & 732 & $125(17.1)$ & 650 & 0 \\
\hline \multicolumn{7}{|l|}{ Age distribution (years) } \\
\hline Less than $1(\mathrm{n}=38)$ & 29 & $7(24.1)$ & 19 & $4(21.1)$ & 24 & 0 \\
\hline $1-5(n=337)$ & 336 & $71(21.1)$ & 165 & $13(7.9)$ & 156 & 0 \\
\hline $6-12(\mathrm{n}=539)$ & 529 & $185(35.0)$ & 233 & $20(8.6)$ & 209 & 0 \\
\hline $13-18(\mathrm{n}=548)$ & 541 & $252(46.6)$ & 170 & $16(9.4)$ & 180 & 0 \\
\hline $19-59(\mathrm{n}=3383)$ & 3328 & $1623(48.8)$ & 1095 & $175(16.0)$ & 1017 & 0 \\
\hline $60 \&$ above $(n=353)$ & 337 & $129(38.3)$ & 134 & $31(23.1)$ & 98 & 0 \\
\hline
\end{tabular}

\begin{tabular}{lcc}
\hline Clinical manifestations & Positive for Dengue $(\%)$ & Positive for Chikungunya (\%) \\
\hline Fever & 99.73 & 100.00 \\
Headache & 33.24 & 16.66 \\
Malaise & 22.25 & 10.00 \\
Myalgia & 26.54 & 13.33 \\
Arthralgia & 64.88 & 80.00 \\
Haemorrhagic manifestations & 8.58 & 6.66 \\
Retro-orbital pain & 8.58 & 3.33 \\
Rigors & 11.52 & 0.00 \\
Chills & 12.33 & 10.00 \\
\hline
\end{tabular}

a potent epidemiological threat throughout the world accounting for the most important emerging and reemerging vector borne viral diseases. Involvement of same vector in disease transmission and rise in the numbers of cases of microcephaly related to Zika virus, chronic joint disease post-chikungunya infection and potentially lethal complication known as severe dengue make the co-circulation of these three diseases a matter of great public health importance. DENV, CHIKV and ZIKV, currently prevalent are responsible for significant epidemics occurring globally [10]. In most parts of India, occurrence of dengue infection is a regular phenomenon with periodic upsurges and also there is a progressive spread of chikungunya virus from south to north Indian regions in the last decade [11, 12]. First dengue outbreak was reported in the state of Odisha in the year 2010 which continued in the subsequent years in the form of epidemic during post monsoon period gradually becoming endemic in nature [13]. India experienced outbreaks due to CHIK infection in 2005 after a long gap [14] whereas Odisha with no history of Chikungunya infection experienced its first epidemic in 2006. A study from Odisha confirmed the emergence of CHIKV infection in Odisha in 2006 and its spread was facilitated by the presence of the Aedes vector species affecting a wide geographical area within a short duration of time [15]. This study is the first to report the prevalence of dengue, re- 
Fig. 2 Seasonal prevalence of dengue/chik suspected and positive cases (2016-2019)

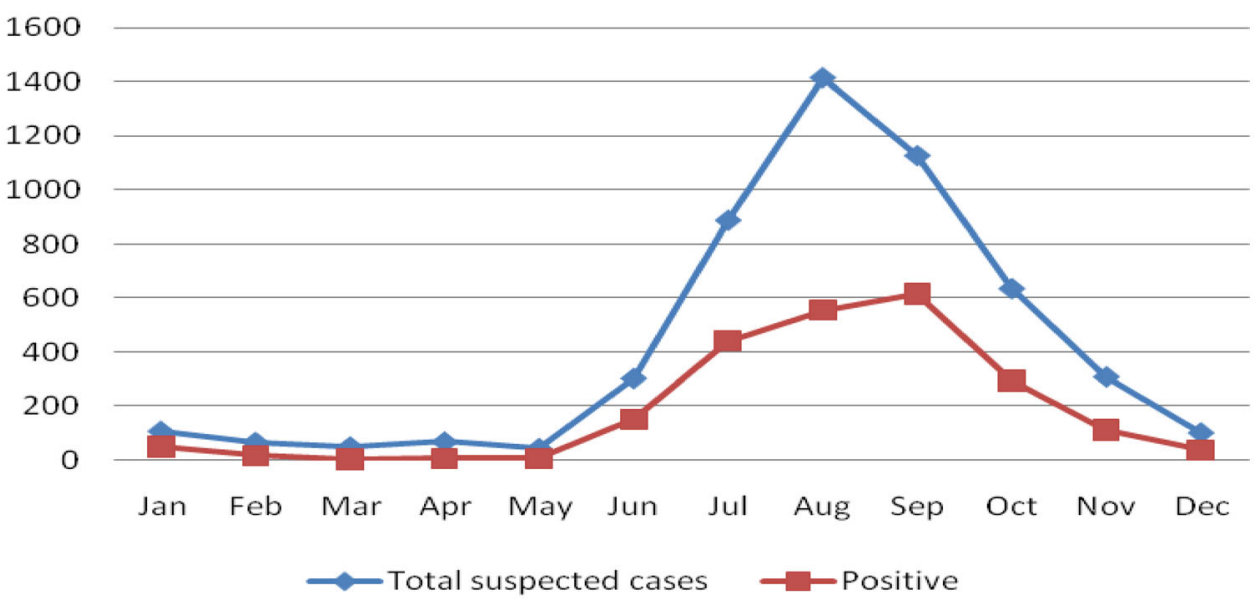

emerging infection chikungunya and globally emerging arboviral infection Zika in the state of Odisha. The results of this study will form a basis to formulate clinical management protocol for affected patients and execute effective control measures for regionally prevalent arboviral infections. It was noted that in majority of cases of dengue, illness was mild in nature indicating the endemicity of the virus in most regions of Odisha and severe dengue cases were associated with mixed infections [13]. In this study, $43.8 \%$ dengue seropositivity was noted which is in concordance with the results reported by Gupta et al. [16]. However in contrast a study from Jammu reported lower dengue seropositivity [17]. Much higher seropositivity rates were also reported from Delhi and Gwalior i.e. 95\% and $65 \%$ respectively $[18,19]$ which can be attributed to huge migration of population from states of India where dengue is endemic. Increased urbanization, inadequate control of mosquito vector, gradually waning herd immunity and A226V mutation of E1 gene of CHIKV genome are factors responsible for consequential rise in CHIKV cases and re-emergence of chikungunya [20]. Around 58,136 cases of chikungunya have been detected across the country in 2016 as per a report from NVBDCP [21]. However, regular dengue outbreaks have been reported in the state of Odisha along with fewer chikungunya outbreaks but no cases positive for ZIKV have been reported till date, despite the favorability of climate of Odisha for the vectors associated with spread of these arboviruses. In our study 110/1729 (6.36\%), CHIKV and DENV co-infection cases was detected. The co-infection is significant and worth considering during clinical management of patients as it contributes to increased morbidity as well as mortality, especially in debilitated, immuno-compromised and older patients. Being transmitted by the same vector i.e. Aedes mosquitoes, both arboviral diseases are often found co-circulating and the occurrence of co-infection is not unusual $[22,23]$. In our study subjects, it was noted that affected cases were mostly males when compared to females. This may be either because males are exposed more to bites of mosquitoes due to their nature of activity which is mostly out door or due to sample biasing in the population caused by gender difference in seeking health care. Hence, it can be very well ruled out that dengue is gender specific disease which is in agreement with the results reported by Sabat et. al. [13]. Season is a significant determining factor in the incidence of infectious diseases, mainly arboviral infections, such as dengue, chikungunya, and Zika. Climatic factors like temperature, humidity and rainfall in addition to human behavioural trends contribute to the seasonal patterns of arboviral diseases [24]. Month wise distribution of dengue and chikungunya cases in this study shown a gradual increase from July month onwards which is in concurrence with other outbreaks reported from India $[25,26]$. In this study the correlation between incidence of arboviral infections and monsoon season was clearly evident and it has been also supported in previous studies [27, 28]. Sudden climate change, urbanization, extensive agriculture, migration of humans and animals, mass gathering events, development of air transport etc. have all contributed to the rapid worldwide spread of arboviral diseases $[14,29]$. Also, it was noted that the load of arboviral diseases was more in coastal areas as compared to non-coastal which may be attributed to the suitability of climatic conditions for the breeding of vector [30]. Clinically, the three arboviral diseases are represented by similar clinical manifestations making it often difficult to differentiate among them. Common clinical symptoms of these three infections included fever, exanthema, conjunctivitis, retro-orbital pain, headache and arthralgia. Similar findings were also reported by Cardoso et al., Azeredo et al. and Solomon et al. [31-33]. Beltrán-Silva $\mathrm{SL}$, et al. reported that for patients with acute fever, myalgia or arthralgia, macula-papular rash and recent travel to endemic areas within the previous 14 days, DENV 
CHIKV, and ZIKV infections should all be taken into consideration [9]. As the clinical course of the disease drastically ranges from a self-limiting, relatively benign disease to a potentially life-threatening one, timely diagnosis and careful monitoring of confirmed patients is of utmost importance [34]. Serotyping of NS1 positive samples of dengue suspected cases revealed circulation of all four serotypes in Odisha which is in concordance with previous reports [35]. The CHIKV strains detected were the East, Central and South African (ECSA) genotype with E1 A226V mutation.

\section{Conclusion}

The emergence of ZIKV, re-emergence of CHIKV and continuous annual occurrence of DENV in India causing high morbidity, unmanageable complications, increasing burden on health care system and other supportive services has led to unprecedented circumstances. This is not trivial because the situation demands for measures for effective prevention and control to protect the susceptible population. Currently there is unavailability of specific treatment or vaccine for control of these arboviral diseases. With the ongoing scenario and the experience with DENV, CHIKV and ZIKV, it is clear that introduction of new pathogens is inevitable in the future leading to new epidemics further weakening the public health system. In order to avoid successive epidemics of arboviral diseases we have to develop better technologies to control mosquito populations. Rapid increase in globalization is causing weakening of traditional barriers that once curtailed the spread of diseases within regions and thus, spreading the diseases to populations lacking native immunity. Clinicians and health care workers are on the front lines of detection of emerging infections and hence, must be vigilant in drawing out a careful clinical and travel history from suspected patients. Prompt recognition and provision of effective treatment of these diseases is the need of the hour for containing outbreaks. The present study clearly shows that dengue is well established and a constant seasonal alarm in this eastern coastal state of India. Chikungunya and its co-infection with dengue is also a matter of raised concern and it is high time that public health officials are aware of the importance of early recognition, diagnosis and prompt clinical management of suspected cases. Zika virus disease is another potential threat looming on the horizon for health care workers as well as researchers not only in India but whole world.

Understanding the disease pattern and general predictions will help in mitigating the effects of changing climate on the emergence and re-emergence of new or existing infections. It's high time to strengthen the current surveillance systems and methods for vector control, as well as on the development of antiviral agents and effective vaccine candidates. Hence, it is of utmost importance, in a region like Odisha which is conducive for vectors responsible for arboviral diseases, for physicians to be familiar with epidemiology of arboviral diseases, wide range of clinical manifestations, and laboratory based diagnostic tools for the accurate diagnosis, revised treatment strategies and prevention of associated complications.

Acknowledgements We thank DHR (ICMR), New Delhi for supporting this work.

Authors' contributions Conceived and designed the study SS, JS, BD, JT; Performed experiment and collected the data SS, JS, SP, MCM; Data analysis SS, JS, BD, JT, SuP; Drafting of manuscript SS; Revision and approval of manuscript-all authors.

Funding DHR, New Delhi.

Data availability Yes, it is available.

\section{Declarations}

Conflict of interest The authors declare no conflict of interest.

Consent to participate The study was approved by Human Ethical Committee of the Institution following guidelines of Indian Council of Medical Research (ICMR), New Delhi.

Consent for publication Not applicable

Ethical Approval Not applicable

\section{References}

1. World Health Organization. Dengue: guidelines for diagnoses, treatment, prevention and control. Geneva: WHO; 2009.

2. Burt FJ, Rolph MS, Rulli NE, et al. Chikungunya: a re-emerging virus. Lancet. 2012;379(662-71):2.

3. Centers for Disease Control and Protection. Chikungunya: Information for Healthcare Providers. Available at: http://www. cdc.gov/chikungunya/pdfs/CHIKV_Clinicians.pdf. Accessed April 10, 2016.

4. Gulland A. Zika virus is a global public health emergency, declares WHO. BMJ. 2016:1657.

5. Patterson J, Sammon M, Garg M. Dengue, Zika and Chikungunya: emerging arboviruses in the new world. West $\mathrm{J}$ Emerg Med. 2016;17(6):671-9. https://doi.org/10.5811/westjem.2016.9. 30904.

6. Ioos S, Mallet HP, Leparc Goffart I, et al. Current Zika virus epidemiology and recent epidemics. Med Mal Infect. 2014;44:302-7.

7. Lanciotti RS, Calisher CH, Gubler DJ, Chang GJ, Vorndam AV. Rapid detection and typing of dengue viruses from clinical samples by using reverse transcriptase-polymerase chain reaction. J Clin Microbiol. 1992;30(3):545-51.

8. Pfeffer M, Linssen B, Parker MD, Kinney RM. Specific detection of chikungunya virus using a RT-PCR/nested PCR combination. J Vet Med B Infect Dis Vet Public Health. 2002;49(1):49-54. 
9. Das B, Sahu A, Das M, Patra A, Dwibedi B, Kar SK, Hazra RK. Molecular investigations of chikungunya virus during outbreaks in Orissa, Eastern India in 2010. Infect Genet Evol. 2012;12(5):1094-101. https://doi.org/10.1016/j.meegid.2012.03. 012.

10. Beltrán-Silva SL, et al. Clinical and differential diagnosis: dengue, chikungunya and Zika. Rev Med Hosp Gen Méx. 2016. https://doi.org/10.1016/j.hgmx.2016.09.011.

11. Singh B. Dengue outbreak in 2006: failure of public health system? Indian J Commun Med. 2006;32:99-100.

12. Nagpal BN, Saxena R, Srivastava A, Singh N, Gosh SK, Sharma SK, et al. Retrospective study of chikungunya outbreak in urban areas of India. Indian J Med Res. 2012;135(3):351-8.

13. Sabat J, Subhadra S, Thakur B, Panda M, Panda S, Pati SS, Ho LM, Dixit S, Rathore SK, Kar SK, Dwibedi B. Molecular and phylogenetic analysis of the dengue strains circulating in Odisha, India. Virus Dis. 2019. https://doi.org/10.1007/s13337-01900544-3.

14. Lahariya C, Pradhan SK. Emergence of chikungunya virus in Indian subcontinent after 32 years: a review. J Vector Borne Dis. 2006;43(4):151-60.

15. Dwibedi B, Sabat J, Mahapatra N, et al. Rapid spread of chikungunya virus infection in Orissa: India. Indian J Med Res. 2011;133(3):316-21.

16. Gupta E, Dar L, Narang P, Srivastava VK, Barror S. Serodiagnosis of dengue during an outbreak at a tertiary hospital in Delhi. Indian J Med Res. 2005;121:36-8.

17. Shashi SS, et al. Serosurveillance of Dengue. Chikungunya and Zika in Jammu, a Sub-Himalayan Region of India, J Clin Diagn Res. 2017;11(11):DC05-8.

18. Kurukumbi M, Wali JP, Broor S, Aggarwal P, Seth P, Handa R, et al. Seroepidemiology and active surveillance of dengue fever/dengue haemorrhagic fever in Delhi. Indian J Med Sci. 2001;55(3):149-56.

19. Parida MM, Dash PK, Upadhyay C, Saxena JAM. Serological and virological investigation of an Outbreak of Dengue fever in Gwalior, India. Indian J Med Res. 2002;116:248-54.

20. Pialoux G, Gaüzère BA, Jauréguiberry S, Strobel M. Chikungunya: an epidemic arbovirosis. Lancet Infect Dis. 2007;7:319-27.

21. National Vector Borne Disease Control Programme. Chikungunya fever. 2017; http://nvbdcp.gov.in/chikun-status.html. Accessed 24 May 2017.

22. Furuya-Kanamori L, Liang S, Milinovich G, Magalhaes RJS, Clements ACA, $\mathrm{Hu} \mathrm{W}$, et al. Co-distribution and co-infection of chikungunya and dengue viruses. BMC Infect Dis. 2016;16:84.

23. Singh J, Dinkar A, Singh RG, Siddiqui MS, Sinha N, Singh SK. Clinical profile of dengue fever and coinfection with chikungunya. Ci Ji Yi Xue Za Zhi. 2018;30(3):158-64. https://doi.org/ 10.4103/tcmj.tcmj_138_17.
24. Sippy R, Herrera D, Gaus D, Gangnon RE, Patz JA, Osorio JE. Seasonal patterns of dengue fever in rural Ecuador: 2009-2016. PLoS Negl Trop Dis. 2019;13(5): e0007360. https://doi.org/10. 1371/journal.pntd.0007360.

25. Kakarla SG, Caminade C, Mutheneni SR, et al. Lag effect of climatic variables on dengue burden in India. Epidemiol Infect. 2019;147: e170. https://doi.org/10.1017/S0950268819000608.

26. Ganeshkumar P, Murhekar MV, Poornima V, Saravanakumar V, Sukumaran K, Anandaselvasankar A, et al. Dengue infection in India: a systematic review and meta-analysis. PLoS Negl Trop Dis. 2018;12(7): e0006618. https://doi.org/10.1371/journal.pntd. 0006618 .

27. Broor S, Devi LS. Arboviral Infections in India. Indian J Health Sci Care. 2015;2(3):192-202.

28. Liang G, Gao X, Gould EA. Factors responsible for the emergence of arboviruses; strategies, challenges and limitations for their control. Emerg Microbes Infect. 2015;4(3): e18. https://doi. org/10.1038/emi.2015.18.

29. Kilpatrick AM, Randolph SE. Drivers, dynamics, and control of emerging vector-borne zoonotic diseases. Lancet. 2012;380:1946-55.

30. Chowell G, Cazelles B, Broutin H, Munayco CV. The influence of geographic and climate factors on the timing of dengue epidemics in Perú, 1994-2008. BMC Infect Dis. 2011;11:164. https://doi.org/10.1186/1471-2334-11-164.

31. Cardoso CW, Paploski IA, Kikuti M, et al. Outbreak of Exanthematous Illness Associated with Zika, Chikungunya, and Dengue Viruses, Salvador, Brazil. Emerg Infect Dis. 2015;21(12):2274-6. https://doi.org/10.3201/eid2112.151167.

32. Azeredo EL, dos Santos FB, Barbosa LS, Souza TMA, BadolatoCorrêa J, Sánchez-Arcila JC, Nunes PCG, de-Oliveira-Pinto LM, de Filippis AM, Dal Fabbro M, Hoscher Romanholi I, Venancio da Cunha R. Clinical and Laboratory Profile of Zika and Dengue Infected Patients: Lessons Learned From the Co-circulation of Dengue, Zika and Chikungunya in Brazil. PLOS Currents Outbreaks. 2018 Feb 15. Edition 1. https://doi.org/10.1371/currents. outbreaks.0bf6aeb4d30824de63c4d5d745b217f5.

33. Solomon $\mathrm{T}$, Dung $\mathrm{N}$, Vaughn $\mathrm{D}$, Kneen $\mathrm{R}$, Thao $\mathrm{L}$, Raengsakulrach B, et al. Neurological manifestations of dengue infection. Lancet. 2000;355:1053-8.

34. Centers for Disease Control and Prevention. Clinical Guidance Dengue Virus. http://www.cdc.gov/dengue/clinicalLab/clinical. html. Accessed 30 Mar 2016.

35. Mishra B, Turuk J, Sahu SJ, Khajuria A, Kumar S, Dey A, Praharaj AK. Co-circulation of all four dengue virus serotypes: first report from Odisha. Indian J Med Microbiol. 2017;35:293-5.

Publisher's Note Springer Nature remains neutral with regard to jurisdictional claims in published maps and institutional affiliations. 